American Journal of Biochemistry and Biotechnology 7 (4): 196-203, 2011

ISSN 1553-3468

(C) 2011 L. Pulakat et al., This open access article is distributed under a Creative Commons Attribution

(CC-BY) 3.0 license

\title{
Discovery of Evolutionary Divergence of Biological Nitrogen Fixation and Photosynthesis: Fine Tuning of Biogenesis of the NifH and the ChlL by a Peptidyl-Prolyl Cis/Trans Isomerase
}

\author{
${ }^{1,2}$ Nara Gavini, ${ }^{1}$ Sinny Delacroix, \\ ${ }^{2}$ Kelvin Harris Jr. and ${ }^{1,2,3}$ Lakshmi Pulakat \\ ${ }^{1}$ Department of Biological Sciences, \\ Bowling Green State University, OH-40342, Bowling Green \\ ${ }^{2}$ Department of Biological Sciences, \\ Mississippi State University, MS-39762, Mississippi State \\ ${ }^{3}$ Department of Internal Medicine, \\ University of Missouri, MO-65212, Columbia
}

\begin{abstract}
Problem statement: Despite the structural and functional similarities between the nitrogenase that performs biological nitrogen fixation reaction and the Dark Protochlorphyllide Oxidoreductase (DPOR) that performs chlorophyll-biosynthesis, attempts to substitute nitrogenasecomponents with DPOR-components have hitherto failed. This investigation was undertaken to test if Chlamydomonas reinhardtii protochlorophyllide (Pchlide) reductase (ChlL) that shares some structural similarity with Nitrogenase Reductase (NifH) could complement the functions of NifH in biological nitrogen fixation of Azotobacter vinelandii. Approach: Genetic complementation studies were performed to test if the $c h l L$ gene and its mutants cloned under transcriptional control of nifH promoter (nifHp) in a broad-host range low copy plasmid pBG1380 could render a $\mathrm{Nif}^{+}$phenotype to NifHdeficient A. vinelandii strains. Results: Expression of ChlL could render $\mathrm{Nif}^{+}$phenotype to NifHdeficient $A$. vinelandii only in the absence of NifM, a nif-specific PPIase essential for biogenesis of NifH. The ChlL mutants Cys95Thr and Cys129Thr were unable to substitute for NifH. Thus, the conserved cysteine ligands of [4Fe-4S] cluster in ChlL are essential for successful substitution of NifH by ChlL. Since C-termini of NifH and ChlL demonstrated the least similarity and Pro258, a substrate for the PPIase activity of NifM, is located in the C-terminus of NifH, we posited that replacing the $\mathrm{C}$-terminus of $\mathrm{NifH}$ with that of $\mathrm{ChlL}$ would render NifM-independence to NifH. The NifH-ChlL chimera could support the growth of NifH- and NifM-deficient A. vinelandii in nitrogen limiting conditions implying that it has acquired NifM-independence. Conclusion/Recommendations: Collectively, these observations suggest that NifM, an evolutionarily conserved nif-specific PPIase, could have contributed to the functional divergence of biological nitrogen fixation and photosynthesis during evolution by virtue of its ability to exert opposing effects on structurally similar substrates, ChlL and NifH.
\end{abstract}

Key words: Nitrogenase, NifH, ChlL, Dark Protochlorphyllide Oxidoreductase (DPOR), NifM, PPIase

\section{INTRODUCTION}

Functional divergence of biological nitrogen fixation and photosynthesis, the two fundamental biological processes that sustain life on earth, is still an enigma. Structural and functional similarities exist between nitrogenase that performs the biological nitrogen fixation reaction and Dark Protochlorphyllide Oxidoreductase (DPOR) that performs reduction of protochlorophyllide to chlorophyllide during chlorophyll-biosynthesis (Brocker et al., 2008; Gavini et al., 2006; Georgiadis et al., 1992; Sarma et al., 2008; Tezcan et al., 2005; Watzlich et al., 2009; Yamamoto et al., 2009; 2008; Yamazaki et al., 2006a; 2006b; Nomata et al., 2006a; 2006b). Both nitrogenase and DPOR are oxygen sensitive twocomponent systems. However, attempts to substitute nitrogenase components NifH or NifDK by DPOR components have not been successful thus far.

\section{Corresponding Author: Lakshmi Pulakat, Department of Internal Medicine, University of Missouri, MO-65212, Columbia} Tel: 573-814-6000 


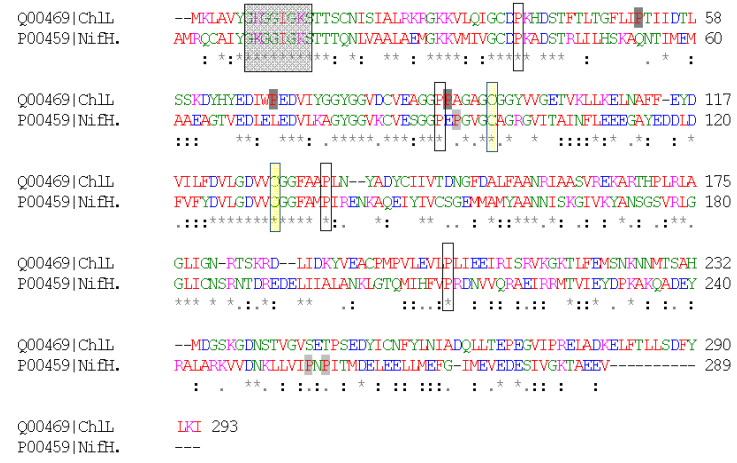

Fig. 1: Comparison of the amino acid sequences of $A$. vinelandii $\mathrm{NifH}$ and $C$. reinhardtii $\mathrm{ChlL}$ using. ClustalW (http://www.clustal.org/). The conserved cysteine ligands are shown in light yellow box. The ATP binding site is shown in gray box. The four proline residues that are conserved between the NifH, BchL and the ChlL are marked by open box. Three more additional proline residues that are conserved among NifH peptides are highlighted in light gray and the additional proline residues that are conserved among BchL and ChlL (shown in Fig. 2) are highlighted in dark gray

Nitrogenase is dependent on a multitude of nif-specific accessory proteins for its maturation and assembly (Brocker et al., 2008; Gavini et al., 2006; Georgiadis et al., 1992; Yamazaki et al., 2006b; Betancourt et al., 2008; Chen et al., 1994; Christiansen et al., 2001; Curatti et al., 2007; Gavini and Burgess, 1992; Gavini et al., 1994; Finan, 2002; Howard et al., 1989; 1986; Howard and Rees, 1996; Jacobson et al., 1989a; 1989b; Lei et al., 1999; 1998; Peters and Szilagyi, 2006; Petrova et al., 2002; Rubio and Ludden, 2005; Robinson et al., 1987). The DPOR component analogous to the nitrogenase component $\mathrm{NifH}$ is the BchL/ChlL protein encoded by the $b c h L / c h l L$ genes of the photosynthetic bacteria.

Biogenesis of functional $\mathrm{NifH}$ is dependent on nif-accessory protein NifM in its natural system or in heterologous system (Finan, 2002; Howard et al., 1986; Jacobson et al., 1989a; Petrova et al., 2002). The NifM is a peptidyl-prolyl cis/trans isomerase (PPIase) belonging to the Parvulin family (Edlich and Fischer, 2006; Rahfeld et al., 1994). We have proposed that the NifM-mediated cis-to trans isomerization of one or more of the seven conserved prolines is needed for the generation of the functional NifH (Finan, 2002).
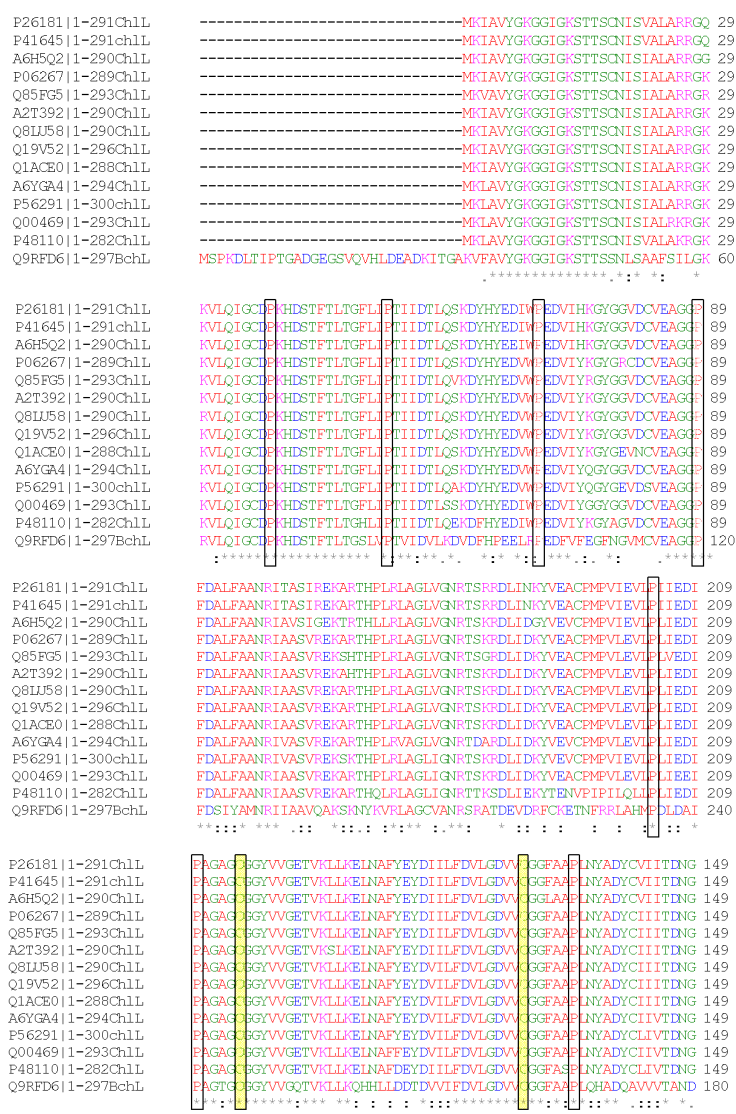

Fig. 2: ClustalW alignment of 13 ChlL peptides with BcHL. The conserved cystein ligands (light yellow box), ATP binding site (gray box) and proline residues (open box ) are marked. P26181: $P$. contorta, P41645: $P$. thunbergii, A6H5Q2: $C$. taitungensis, P06267: M. polymorpha, Q85FG5: A. capillus-veneris, A2T392: A. evecta, Q8LU58: C. globosum, Q19V52: C.atmophyticus, Q1ACE0: C. vulgaris, A6YGA4: L. terrestris, P56291: Chlorella vulgaris, Q00469: $C$. reinhardtii, P48110: C. paradoxa, Q9RFD6: $R$. sphaeroides

Other PPIases are unable to substitute for NifM unless their catalytic domain is fused to the N-terminal region of NifM. A comparison of ChlL with $\mathrm{NifH}$ shows four conserved prolines (Fig. 1) that are part of the nine conserved prolines among the ChlL peptides (Fig. 2). Additionally, superimposing a predicted model of ChlL onto the NifH template (PDB ID: 1NIP) (Georgiadis et al.,1992), using Swiss PDB (Deep View) protein modeling software (Fig. 3) shows that the ChlL protein model thus generated has marked resemblance to the NifH protein. 
Am. J. Biochem. \& Biotech., 7 (4): 196-203, 2011

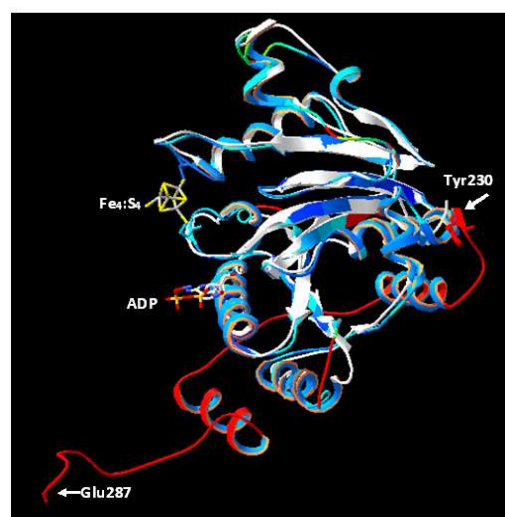

Fig. 3: The NifH monomer structure (based on 1NIP crystallographic structure of the NifH peptide from A. vinelandii (Georgiadis et al., 1992) colored according to differences with the ChlL model. Deep View color for ChlL was white. The Deep View coloration of $\mathrm{NifH}$ was done according to root mean square deviation type of coloration (blue $=$ maximum similarity; red $=$ maximum dissimilarity) in comparison to ChlL. Bottom view shows a particularly dissimilar C-terminal region (red stretch) starting with Tyr230 and ending with Glu287 of NifH. The [4Fe-4S] cluster and the bound $\mathrm{MgADP}$ in NifH are indicated

Since the ChlL protein showed such high structural similarity to the NifH protein, we hypothesized that ChlL would substitute for NifH in A. vinelandii and that NifM may play a role in modulating the functional properties of the NifH-like proteins such as ChlL/Bchl. The purpose of this study was to determine whether ChlL could substitute for NifH in biological nitrogen fixation in presence and absence of functional NifM.

\section{MATERIALS AND METHODS}

Construction of plasmid pBG2400: An 879bp DNA fragment encoding the ORF of chlL flanked by EcoRV and HindIII restriction enzyme sites was generated by PCR amplification using $C$. Reinhardtii chromosomal DNA as template and cloned into the pCR2.1 TOPO vector to create plasmid pBG1382 (Suh, 2002). This fragment was then subcloned into the EcoRV and HindIII digested broad-host range expression vector pBG1380 that contained the nifHp (Gavini et al., 2006) to generate pBG2400. Thus, the plasmid pBG2400 had the chlL gene under the transcriptional regulation of the nifHp.

Construction of ChlL mutants: PCR-mutagenesis with 'QuikChange ${ }^{\mathrm{TM}}$ Site Directed Mutagenesis Kit'
(Stratagene Products, La Jolla, CA) was used to generate these mutants according to manufacturer's protocol. Plasmid pBG2400 was used as template. To generate Cys95Thr amino acid replacement, the forward primer was: 5'CCACCTGCCGGTGCGGGGACCGGTGGTTATGTTG TAGGT-3' and reverse primer was 5'ACCTACAACATAACCACCGGTCCCCGCACCGGC AGGTGG-3'. To generate Cys129Thr amino acid replacement, the forward primer was: 5'GTTTTAGGTGATGTTGTTACCGGTGGCTTTGCTG CTCCA-3' and reverse primer was 5'TGGAGCAGCAAAGCCACCGGTAACAACATCACC TAAAAC-3'.

Construction of NifH-ChlL chimera: The 873bp DNA fragment encoding nifH ORF was PCR amplified using pDB6 (Jacobson et al., 1989b) as the template and initially cloned into the pCR2.1 TOPO vector. The EcoRV- HindIII fragment encoding nifH ORF was subcolned into EcoRV- HindIII digested pBG1380 to generate pBG2434 that carries nifH gene under the transcriptional control of nifH promoter (nifHp). The $129 \mathrm{bp}$ region that carries the last 42 amino acids at the C-terminus of $\mathrm{NifH}$ was removed via Sall digestion. Next, the DNA containing the last 55 codons of the chlL was PCR-amplified using a 5' primer that carries a SalI site (5'GTCGACAATTCTACAGTAGGAGTGTC-3') and a 3' primer with a HindIII site (5AAGCTTTTAAATTTTAAGATAGAAATC-3'). The resultant PCR product encoding the $\mathrm{C}$-terminal region of ChlL protein (55 amino acids at the C-terminal end) was cloned into the SalI-HindIII digested pBG2434 (carrying the N-terminus of nifH) to generate a nifH-chlL chimeric gene in which the DNA encoding the C-terminal region of NifH (bp745-873) was replaced by the DNA encoding C-terminal region of ChlL (bp718-882).

\section{RESULTS AND DISCUSSION}

ChlL can substitutes for $\mathrm{NifH}$ in biological nitrogen fixation reaction only in the absence of NifM: Two NifH-deficient A. vinelandii strains, one NifMpositive (nifM ${ }^{+}$A. vinelandii DJ54 (Gavini et al., 1994; Robinson et al., 1987) and one NifM-negative (nifM::kan A. vinelandii BG98 (Gavini et al., 2006) respectively, were used to test the ability of the ChlL to substitute for the $\mathrm{NifH}$ in nitrogen fixation reaction by A. vinelandii. Both strains were transformed with pBG2400 that carries the $C$. reinhardtii chlL gene cloned under the transcriptional regulation of the nifHp and the ability of the transformants to grow under nitrogen limiting conditions was assessed as follows. 
Am. J. Biochem. \& Biotech., 7 (4): 196-203, 2011

Table 1: Replacing the cysteine ligands Cys95 and Cys129 with Thr inhibits the ability of the ChlL to substitute for the NifH in NifH-deficient NifM-minus A. vinealndii BG98

\begin{tabular}{|c|c|c|c|c|c|}
\hline Strain & Plasmid & $\begin{array}{l}\text { Codon/amino } \\
\text { acid for position } \\
95 \text { of ChlL }\end{array}$ & $\begin{array}{l}\text { Codon/ amino } \\
\text { acid for position } \\
129 \text { of ChlL }\end{array}$ & $\begin{array}{l}\text { Growth on } \mathrm{BN}^{+} \\
\text {medium with } \\
\text { Chloramphenicol }\end{array}$ & $\begin{array}{l}\text { Growth on } \\
\mathrm{BN}^{-} \text {medium }\end{array}$ \\
\hline A. vinelandii DJ54 (AnifH) $\mathrm{NifM}^{+}$ & pBG2400 & TGT/Cys & TGT/Cys & +++ & --- \\
\hline A. vinelandii BG98 nifM $\mathrm{NifH}$-deficient & pBG2400 & TGT/Cys & TGT/Cys & +++ & +++ \\
\hline A. vinelandii BG98 nifM NifH-deficient & pBG2406 & $\mathrm{ACC} / \mathrm{Thr}$ & TGT/Cys & +++ & --- \\
\hline A. vinelandii BG98 nifM NifH-deficient & pBG2428 & TGT/Cys & $\mathrm{ACC} / \mathrm{Thr}$ & +++ & --- \\
\hline A. vinelandii DJ54 (AnifH) $\mathrm{NifM}^{+}$ & pBG2406 & ACC/Thr & TGT/Cys & +++ & --- \\
\hline A. vinelandii DJ54 (AnifH) $\mathrm{NifM}^{+}$ & pBG2428 & TGT/Cys & $\mathrm{ACC} / \mathrm{Thr}$ & +++ & -- \\
\hline
\end{tabular}

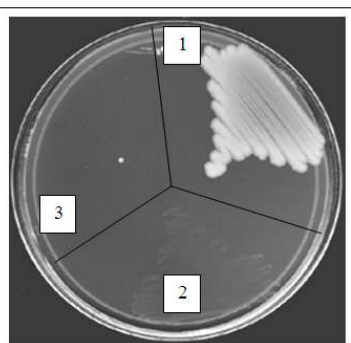

(A)

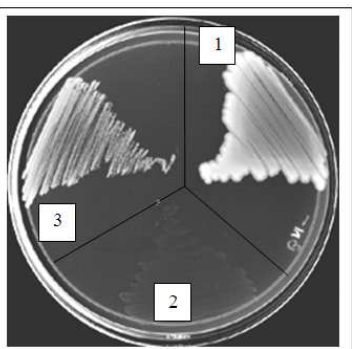

(B)
Fig. 4: Comparative growth of NifH-deficient $A$. vinelandii strains carrying plasmids with or without chlL gene on Burk's nitrogen free $\left(\mathrm{BN}^{-}\right)$ agar plate is shown. A) nifM ${ }^{+}$DnifH A. vinelandii DJ54 carrying pBG1380 that does not harbor chlL (marked 2) or pBG2400 that harbors chlL (marked 3); B) nifM (nifM gene interrupted by insertion of $\operatorname{Kan}^{R}$ ) A. vinelandii $B G 98$ that expresses a non-functional NIfH and harboring pBG1380 (marked 2) or pBG2400 (marked 3). Wild type $A$. vinelandii was used as control (marked as 1) on both plates. The pBG1380 is a parental plasmid that harbors the nifH promoter ${ }^{[2]}$. The pBG2400 carries the chlL under the transcriptional control of the nifHp of pBG1380, therefore, the $c h l L$ is expressed under nitrogen limiting conditions. The presence of pBG2400 allowed the growth of NifH-deficient nifM::kan A. vinelandii $\mathrm{BG} 98$, on $\mathrm{BN}^{-}$agar plates (B 3), while it did not support the growth of NifH-deficient nifM ${ }^{+} A$. vinelandii DJ54 on $\mathrm{BN}^{-}$agar (A 3). Transformations were repeated at least six times

Since the plasmid carries a chloramphenicol resistance $\left(\mathrm{Cm}^{R}\right)$ marker, the transformants were originally selected on Burk's Nitrogen plus $\left(\mathrm{BN}^{+}\right)$(Strandberg and Wilson, 1968) medium supplemented with $72 \mu \mathrm{g}$ $\mathrm{mL}^{-1}$ of chloramphenicol. Then, the ability of the chlL gene to render $\mathrm{Nif}^{+}$phenotype to A. vinelandii strains DJ54 and BG98 was monitored by growing the transformants on Burk's nitrogen free $\left(\mathrm{BN}^{-}\right)$media containing molybdenum
(Strandberg and Wilson, 1968) that imits expression of alternate nitrogenases (Betancourt et al., 2008).

It was found that pBG2400 could not render a $\mathrm{Nif}^{+}$ phenotype to the A. vinelandii DJ54 (Fig. 4a), but it rendered $\mathrm{Nif}^{+}$phenotype to the A. vinelandii BG98 (Fig. 4b)

Since the difference between the two strains is that DJ54 has an intact nifM, whereas BG98 has a disrupted nifM, we concluded that the ChlL could restore nitrogenase activity in the absence of functional NifHbut only if NifM was also absent. In summary, the ChlL can replace the $\mathrm{NifH}$-function in biological nitrogen fixation by $A$. vinelandii. However, the NifM, the accessory PPIase essential for biogenesis of functional $\mathrm{NifH}$, has a negative effect on the compensatory ability of ChlL. This interpretation is consistent with the observations that (a) the chlL gene could not complement the $\Delta$ nifH of nifM $M^{+}$A. vinelandii DJ54 and (b) the BchL protein (similar in structure and function to the ChlL) isolated from nif $M^{+} A$. vinelandii was unable to substitute for the NifH protein in nitrogenase assay (Sarma et al., 2008).

Cys95 and Cys129 of the ChlL are required for substitution of NifH by ChlL in biological nitrogen fixation reaction: The cysteine ligands Cys97 and Cys132 of the NifH peptide are conserved in the ChlL peptide (Cys95 and Cys129 of the ChlL peptide respectively; Fig. 1). Replacing these conserved cysteines of NifH impairs its function (Howard et al., 1989). We posited that the Cys 95 and Cys 129 of the ChlL have a similar role in the ability of the ChlL to participate in nitrogen fixation reaction. Therefore we generated ChlL mutants Cys95Thr and Cys129Thr. In both mutants a TGT to ACC conversion was made that resulted in the codon change TGT (Cys) to ACC (Thr) and created a new PinA1 restriction enzyme site that facilitated identification of these mutants. Locations of the mutations were confirmed by nucleotide sequencing. As shown in Table 1, neither of the mutant chlL genes was able to support the growth of nif $M^{-} \mathrm{NifH}$-deficient $A$. vinelandii $\mathrm{BG} 98$ on $\mathrm{BN}^{-}$medium. 
Am. J. Biochem. \& Biotech., 7 (4): 196-203, 2011
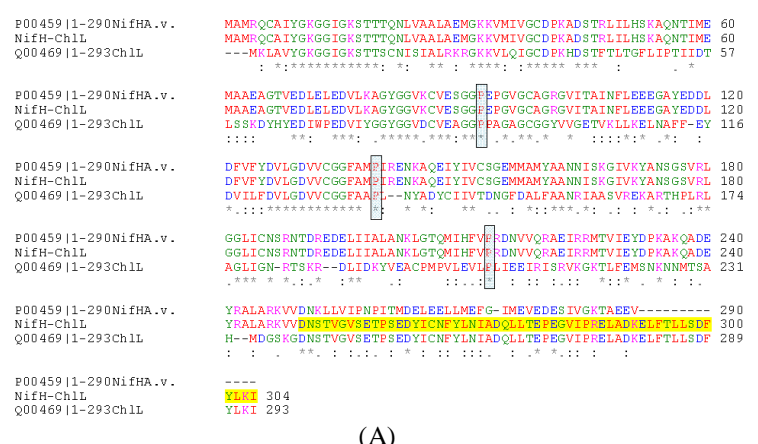

(A)

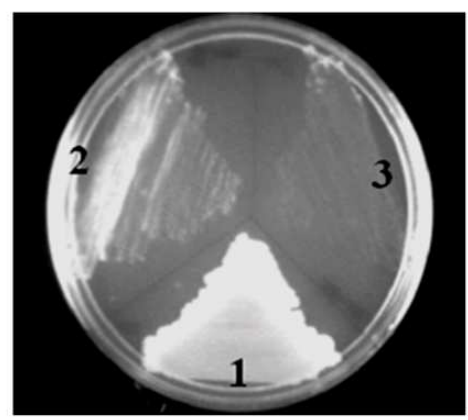

(B)

Fig. 5: (A) Comparison of the amino acid sequence of the NifH-ChlL chimera with the NifH and the ChlL. Conserved proline residues are marked by the open box. The C-terminal region of the $\mathrm{NifH}-\mathrm{ChlL}$ chimera that corresponds to that of the ChlL peptide is highlighted in yellow. (B) Growth of the NifH-deficient nifM A. vinelandii strain BG98 carrying the parental plasmid pBG1380 (marked 2) and pBG1380-derivative expressing the nifH-chlL chimeric gene (marked 3) on $\mathrm{BN}^{-}$medium is shown. Wild type $A$. vinelandii (marked 1) served as control. Thus, the NifH-ChlL chimera could support growth of nifM A. vinelandii strain BG98 in nitrogen limiting conditions indicating that replacement of the Cterminal region of the NifH with that of the ChlL resulted in partial relief from NifM-dependence. Experiments were repeated at least six times

These observations suggested that the Cys95 and Cys129 of the ChlL could play roles analogous to that of Cys97 and Cys132 of $\mathrm{NifH}$ in stabilizing the $[4 \mathrm{Fe}-4 \mathrm{~S}]$ cluster of ChlL.

A nifH-chlL chimera could render $\mathrm{Nif}^{+}$phenotype to A. vinelandii BG98: As shown in Fig. 1 and 3, the Ctermini of the NifH and the ChlL are highly dissimilar. Therefore, the fact that $\mathrm{NifH}$ is not functional in the absence of NifM while ChlL is not functional in the presence of NifM might be traced to this region. We have shown previously that the Pro258 located in the $\mathrm{C}$-terminus of the $\mathrm{NifH}$ is one of the substrates for the PPIase activity of NifM (Gavini et al., 2006).

Because the C-terminus of the ChlL is dissimilar to that of the NifH and the ChlL could substitute the NifH in the absence of the NifM in nitrogen fixation, it is conceivable that the C-terminus of the ChlL would render NifM-independence to the NifH. To test this idea, we analyzed the effect of replacing the $\mathrm{C}$-terminus of NifH with that of ChlL. The DNA encoding the Cterminal region of $\mathrm{NifH}$ (bp745-873) was replaced by the DNA encoding C-terminal region of ChlL (bp718882) to construct the nifH-chlL chimeric gene. Therefore, the resulting NifH-ChlL chimera did not contain Pro258 of the NifH. Amino acid sequence of the NifH-ChlL chimera is shown in Fig. 5a. A. vinelandii BG98 transformants expressing the nifH-chlL chimeric gene were capable of growing on $\mathrm{BN}^{-}$medium (Fig. 5b). Thus, the nifH-chlL chimera could render partial NifMindependence to A. vinelandii BG98.

\section{CONCLUSION}

Our results show that the NifM, a NifH-specific PPIase that is essential for biogenesis of the $\mathrm{NifH}$ protein, has a role in disabling structurally similar ChlL from participating in the biological nitrogen fixation reaction. Significance of PPIase-substrate interactions are particularly highlighted in many pathological conditions. For example, overexpression of human Pin 1 is implicated in the formation of Lewy bodies in Parkinson's Disease, while the same protein has a beneficial effect in Alzheimer's disease, since it regulates amyloid precursor protein processing and amyloid beta production (Pastorino et al., 2006; Ryo et al., 2006). Similarly, Macrophage Infectivity Potentiators (MIPs) are PPIases expressed by bacterial pathogens, however, they interact with host-cell proteins and alter their functions to establish infection (Kohler et al., 2003). These examples show that the molecular interactions between PPIases and proteins that share structural similarity to their natural substrates result in pathogenesis. The example that has emerged from this study is that of a PPIase which could have contributed to the functional divergence of two fundamental biological processes (nitrogen fixation and photosynthesis) during evolution. This is because this PPIase prototype (NifM) would render functionality to one substrate (NifH) and hinder the function of the other structurally similar substrate (ChlL) so that 
nitrogen fixation is favored under conditions that lead to NifM expression (such as nitrogen limitation). These findings represent a unique example of an accessory protein playing a vital part in the evolutionary divergence of biological processes.

The observation that ChlL mutants Cys 95Thr and Cys 129Thr were unable to substitute for NifH further extends the structure-function similarity of the NifH and ChlL related to their mechanistic involvement in nitrogen fixation. Although the structure of ChlL is not yet solved, these observations strengthen the similarities in the role of Cys ligands of the (4Fe-4S) cluster of ChlL in electron transfer by the ChlL to that of the NifH. On the other hand, our studies also highlight the dissimilarity of the C-termini of the NifH and the ChlL. The C-terminus of the NifH is involved in the NifM-dependence of the NifH due to the presence of Pro258 (Gavini et al., 2002). In contrast, the C-terminus of the ChlL could render NifMindependence to the $\mathrm{NifH}$ as shown by the functional NIfH-ChlL chimera (Fig. 5). It is conceivable that a protein similar to the NifH-ChlL chimera could have served as a common ancestor for the NifH and the ChlL before the functional divergence of biological nitrogen fixation and photosynthesis during evolution.

\section{ACKNOWLEDGMENT}

We thank members of Gavini/Pulakat laboratories for valuable comments and technical help. Funding for this research was provided by NSF grant \# MCB1041718 to LP.

\section{REFERENCES}

Betancourt, D.A., T.M. Loveless, J.W. Brown and P.E. Bishop, 2008. Characterization of diazotrophs containing Mo-independent nitrogenases, isolated from diverse natural environments. Appl. Environ. Microbiol., 74: 3471-3480. PMID: 18378646

Brocker, M.J., D. Watzlich, F. Uliczka, M. Saggu and F. Lendzian et al., 2008. Substrate recognition of nitrogenase-like dark operative protochlorophyllide oxidoreductase from Prochlorococcus marinus. J. Biol. Chem., 283: 29873-29881. PMID: 18693243

Chen, L., N. Gavini, H. Tsuruta, D. Eliezer and B.K. Burgess et al., 1994. MgATP-induced conformational changes in the iron protein from Azotobacter vinelandii, as studied by small-angle x-ray scattering. J. Biol. Chem., 269: 3290-3294. PMID: 8106367

Christiansen, J., D.R. Dean and L.C. Seefeldt, 2001. Mechanistic features of Mo-containing Nitrogenase. Annu. Rev. Plant. Physiology. Plant. Mol. Biol., 52: 269-295. PMID: 11337399
Curatti, L., J.A. Hernandez, R.Y. Igarashi, B. Soboh and D. Zhao et al., 2007. In vitro synthesis of the iron-molybdenum cofactor of nitrogenase from iron, sulfur, molybdenum and homocitrate using purified proteins. Proc. Natl. Acad. Sci. U S A., 104: 17626-17631. PMID: 17978192

Edlich, F. and G. Fischer, 2006. Pharmacological targeting of catalyzed protein folding: the example of peptide bond cis/trans isomerases. Handb. Exp. Pharmacol., 172: 359-404. PMID: 16610367

Finan, T.M., 2002. Nitrogen Fixation: Global Perspectives. 1st Edn., CABI, Oxford, ISBN: 0851995918, pp: 553.

Gavini, N. and B.K. Burgess, 1992. FeMo cofactor synthesis by a nifH mutant with altered MgATP reactivity. J. Biol. Chem., 267: 21179-21186. PMID: 1400428

Gavini, N., L. Ma, G. Watt and B.K. Burgess, 1994. Purification and characterization of a FeMo cofactor-deficient MoFe protein. Biochemistry., 33: 11842-11849. PMID: 7918402

Gavini, N., N.S. Tungtur and L. Pulakat, 2006. Peptidyl-prolyl cis/trans isomerase-independent functional NifH mutant of Azotobacter vinelandii. J. Bacteriol., 188: 6020-6025. PMID: 16885471

Georgiadis, M.M., H. Komiva, P. Chakrabarti, D. Woo and J.J. Kornuc et al., 1992. Crystallographic structure of the nitrogenase iron protein from Azotobacter vinelandii. Science, 257: 1653-1659. PMID: 1529353

Howard, J.B. and D.C. Rees, 1996. Structural basis of biological nitrogen fixation. Chem. Rev., 96: 2965982. PMID: 11848848

Howard, J.B., R. Davis, B. Moldenhauer, V. L. Cash and D. Dean et al., 1989. Fe: S cluster ligands are the only cysteines required for nitrogenase $\mathrm{Fe}$ protein activities. J. Biol. Chem., 264: 1127011274. PMID: 2500438

Howard, K.S., P.A. McLean, F.B. Hansen, P.V. Lemley and K.S. Koblan et al., 1986. Klebsiella pneumonia nifM gene product is required for stabilization and activation of nitrogenase iron protein in Escherichia coli. J. Biol. Chem., 261: 772-778. PMID: 3001082

Jacobson, M.R., K.E. Brigle, L.T. Bennett, R.A. Setterquist and M.S. Wilson et al., 1989a. Physical and genetic map of the major nif gene cluster from Azotobacter vinelandii. J. Bacteriol., 171: 10171027. PMID: 2644218

Jacobson, M.R., V.L. Cash, M.C. Weiss, N.F. Laird and W. E. Newton et al., 1989b. Biochemical and genetic analysis of the nifUSVWZM cluster from Azotobacter vinelandii. Mol. Gen. Genet., 219: 4957. PMID: 2615765 
Kohler, R.., J. Fanghanel, B. Konig, E. Lüneberg and M. Frosch et al., 2003. Biochemical and functional analysis of the Mip protein: Influence of the $\mathrm{N}$ terminal half and of peptidyl/prolyl isomerase activity on the virulence of Legionella pneumophila. Infect. Immun., 71: 4389-4397. PMID: 12874317

Lei, S., L. Pulakat and N. Gavini, 1999. Regulated expression of the nifM of Azotobacter vinelandii in response to molybdenum and vanadium supplements in Burk's nitrogen-free growth medium. Biochem. Biophys. Res. Commun., 264: 186-190. PMID: 10527862

Lei, S.H., L. Pulakat, K.C. Parker and N. Gavini, 1998. Genetic analysis on the NifW by utilizing the yeast two-hybrid system revealed that the NifW of Azotobacter vinelandii interacts with the NifZ to form higher-order complexes. Biochem. Biophys. Res. Commun., 244: 498-504. PMID: 9514861

Nomata, J., M. Kitashima, K. Inoue and Y. Fujita, 2006a. Nitrogenase Fe protein-like Fe-S cluster is conserved in L-protein (BchL) of dark-operative protochlorophyllide reductase from Rhodobacter capsulatus. FEBS. Lett., 580: 6151-6154. PMID: 17064695

Nomata, J., T. Mizoguchi, H. Tamiaki and Y. Fujita, 2006b. A second nitrogenase-like enzyme for bacteriochlorophyll biosynthesis: reconstitution of chlorophyllide a reductase with purified X-protein (BchX) and YZ-protein (BchY-BchZ) from Rhodobacter capsulatus. J. Biol. Chem., 281: 15021-15028. PMID: 16571720

Pastorino, L., A. Sun, P.J. Lu, X.Z. Zhou and M. Balastik et al., 2006. The prolyl isomerase Pin1 regulates amyloid precursor protein processing and amyloid-beta production. Nature, 44: 528-534. PMID: 16554819

Peters, J.W. and R.K. Szilagyi, 2006. Exploring new frontiers of nitrogenase structure and mechanism. Curr. Opin. Chem. Biol., 2: 101-108. PMID: 16510305

Petrova, N., L. Gigova and P. Venkov, 2002. Dimerization of Rhizobium meliloti NifH protein in Saccharomyces cerevisiae cells requires simultaneous expression of NifM protein. Int. J. Biochem. Cell. Biol., 34: 33-42. PMID: 11733183

Rahfeld, J.U., K.P. Rucknagel, B. Schelbert, B. Ludwig and J. Hacker et al., 1994. Confirmation of the existence of a third family among peptidyl-prolyl cis/trans isomerases. Amino acid sequence and recombinant production of parvulin. FEBS. Lett., 352: 180-184. PMID: 7925971
Robinson, A.C., D.R. Dean and B.K. Burgess, 1987. Iron-molybdenum cofactor biosynthesis in Azotobacter vinelandii requires the iron protein of nitrogenase. J. Biol. Chem., 262: 14327-14332. PMID:3477546

Rubio, L.M. and P.W. Ludden, 2005. Maturation of nitrogenase: A biochemical puzzle. J. Bacteriol., 187: 405-414. PMID: 15629911

Ryo, A., T. Togo, T. Nakai, A. Hirai and M. Nishi et al., 2006. Prolyl-isomerase Pin1 accumulates in lewy bodies of parkinson disease and facilitates formation of alpha-synuclein inclusions. J. Biol. Chem., 281: 4117-4125. PMID: 16365047

Sarma, R., B.M. Barney, T.L. Hamilton, A. Jones and L.C. Seefeldt et al., 2008. Crystal structure of the L protein of Rhodobacter sphaeroides lightindependent protochlorophyllide reductase with MgADP bound: A homologue of the nitrogenase Fe protein. Biochemistry, 47: 13004-13015. PMID: 19006326

Strandberg, G.W. and P.W. Wilson, 1968. Formation of the nitrogen-fixing enzyme system in Azotobacter vinelandii. Can. J. Microbiol., 14: 25-31. PMID:5644401

Suh, M.H., 2002. Engineering a simplified functional nitrogenase by gene fusion and DNA shuffling. Doctoral Dissertation, Bowling Green State University.

Tezcan, F.A., J.T. Kaiser, D. Mustafi, M.Y. Walton and J.B. Howard et al., 2005. Nitrogenase complexes: Multiple docking sites for a nucleotide switch protein. Science, 309: 1377-1380. PMID: 16123301

Watzlich, D., M.J. Brocker, F. Uliczka, M. Ribbe and S. Virus et al., 2009. Chimeric nitrogenaselike enzymes of (bacterio)chlorophyll biosynthesis. J. Biol. Chem., 284: 15530-15540. PMID: 19336405

Yamamoto, H., J. Nomata and Y. Fujita, 2008. Functional expression of nitrogenase-like protochlorophyllide reductase from Rhodobacter capsulatus in Escherichia coli. Photochem. Photobiol. Sci., 7: 1238-1242. PMID: 18846289

Yamamoto, H., S. Kurumiya, R. Ohashi, Y. Fujita, 2009. Oxygen sensitivity of a nitrogenaselike protochlorophyllide reductase from the cyanobacterium Leptolyngbya boryana. Plant. Cell. Physiol., 50: 1663-1673. PMID: 19643808 
Am. J. Biochem. \& Biotech., 7 (4): 196-203, 2011

Yamazaki, S., J. Nomata and Y. Fujita, 2006a. Differential operation of dual protochlorophyllide reductases for chlorophyll biosynthesis in response to environmental oxygen levels in the cyanobacterium Leptolyngbya boryana. Plant. Physiol., 142: 911-922. PMID: 17028153
Yamazaki, S., J. Nomata and Y. Fujita, 2006b. Nitrogenase $\mathrm{Fe}$ protein-like $\mathrm{Fe}-\mathrm{S}$ cluster is conserved in L-protein (BchL) of dark-operative protochlorophyllide reductase from Rhodobacter capsulatus. FEBS. Lett., 580: 6151-6154. PMID: 17064695 